\section{Risks and benefits of (pharmaco)epidemiology}

\author{
Lesley Wise
}

\begin{abstract}
Pharmacoepidemiology is a relatively young scientific discipline that has seen tremendous growth in interest over the past two decades. This is partly due to changes in the approval of new drugs, which often now require proactive studies to monitor and extend the knowledge of safety in the postauthorization period. Pharmacoepidemiology studies are observational in nature, in contrast to the randomized controlled trial which is the mainstay of drug development. This means that such studies need careful design and execution in order to maximize the benefits of proactive research in the 'real-life' clinical environment and minimize the risks of false results.
\end{abstract}

Keywords: benefit, bias, confounding, pharmaceutical, pharmacoepidemiology, postauthorization, risk

\section{Introduction}

Pharmacoepidemiology is a relatively young scientific discipline which effectively bridges two much older sciences - pharmacology and epidemiology - and borrows heavily from each of these. The International Society for Pharmacoepidemiology [ISPE, 2010] recently held its 26th Annual Meeting in Brighton UK (August 2010), and the author noticed strong anecdotal evidence of the rapid growth in pharmacoepidemiology based on comparing the attendance at a similar conference 8 years ago, held in Edinburgh, Scotland in August 2002. This growth has been seen in most regions of the world and has been driven by a number of factors, such as an increasing awareness of the need to be more proactive in pharmacovigilance, the need for a better understanding of the target population for medicines, the need to provide outcome data particularly on safety endpoints, and the awareness of academic statisticians/epidemiologists of the need for developments in methodology in this field. Some of this has been related to a number of high-profile drug safety issues (such as selective serotonin receptor inhibitors, cyclooxygenase [COX] II inhibitors and rosiglitazone) coupled with an increasing awareness of the limitations of drug safety data from clinical trials and postauthorization spontaneous reports of safety concerns. The development of the International Conference on Harmonisation guideline for Pharmacovigilance Planning (ICH $\mathrm{E} 2 \mathrm{E})[\mathrm{ICH}, 2010]$ in particular resulted in increased use of pharmacoepidemiology within the pharmaceutical industry, as different regions of the world began to request more proactive planning to deal with pharmacovigilance (European Union [EU] and Japan), and risk minimization (EU and USA).

Pharmacoepidemiology is a fascinating branch of epidemiology where it can be reasonably argued that confounding may be more important and difficult to control than in many other branches of the discipline. This is because there are often many factors that affect the specific treatment a patient is given, which may also affect their outcome from the treatment and the disease being treated. This is in contrast to randomized controlled studies, when treatment allocation is decided by random allocation, and provided the study is large enough, and conducted in line with good practice, most of these factors should be balanced between treatment groups.

Consequently, within certain circles pharmacoepidemiology studies have been treated with caution or even disbelief. This article will try to establish how real these concerns are, and the optimal role that pharmacoepidemiology can play in outcomes and pharmacovigilance research, by considering the benefits and risks of the use of pharmacoepidemiology in the drug development and postapproval process. The article does not set out to develop guidance on what study design to use in which situation, which is
Ther Adv Drug Saf (2011) 2(3) 95-102 DOI: $10.1177 /$ 2042098611404920 (C) The Author(s), 2011. Reprints and permissions: http://www.sagepub.co.uk/ journalsPermissions.nav

Correspondence to: Lesley Wise Takeda Global Research and Development (Europe), 61 Aldwych, London WC2B 4AE, UK l.wiseatgrd.com 
definitely beyond the scope of a single article, although reference to some study methodologies will be made in addressing some of the risks and benefits of observational research. Pharmacoepidemiology borrows from pharmacology and epidemiology and the relative importance of the two disciplines in the study design varies according to the stage of the drug development process.

\section{Sources of data}

One major consideration on the conduct of pharmacoepidemiology studies either pre- or postauthorization is the availability of data. There are two basic possibilities, each with their own advantages and drawbacks. Firstly, using data already collected for other purposes. Electronic health record databases (e.g. PHARMO [PHARMO, 2010], General Practice Research Database - GPRD [GPRD, 2010]) have proved to be an invaluable resource for such studies because these often contain prescribing, underlying disease, demographic and outcome information. Other database models also exist which allow for linking of data via a unique number, or are maintained by health insurance companies. These databases can usually provide a wealth of data on comorbidities, but care needs to be taken because the data were not collected for the purposes of research. Therefore, important information may be missing, and this may not necessarily be random. For example, consider the potential primary health record of a patient with angina. This is likely to record smoking status as well as information on a range of other potential confounders. However, such data may not necessarily be recorded for a potential control patient, which will then lead to inaccurate results from any studies. Similarly, health insurance membership (and therefore databases maintained by health insurance companies) may be restricted to people with less underlying serious disease, or predominantly people in employment. Consequently, studies in such databases may not reflect the breadth of exposure in the target population and any impact of adverse effects that may be differential according to underlying disease status may not be detected.

The second option is to follow a cohort of patients and collect specific research quality data on these patients. However, this is expensive and difficult. Preauthorization, these studies would necessitate some form of disease registry. Postauthorization there may be a need to recruit prescribing physicians who may be too busy or unwilling to participate, particularly if they can prescribe the product without needing to be part of the study. Additionally, simply by being in a study the outcomes will be less related to 'real life' than normal clinical practice, and there is likely to be a certain amount of selection bias if people can choose whether or not to participate in the study (see below for further discussion on the potential impact of observational studies and the use of routine data).

\section{Pharmacoepidemiology in drug development}

The many factors that may affect both exposure and response to drug therapy mean that the mainstay of drug development has always been the randomized controlled trial, preferably double blinded. These randomized, blinded designs reduce the impact of known and unknown confounding factors, both those affecting the physician's decision to prescribe and those affecting patient response, to ensure that different treatment groups are as similar as possible at baseline, so that the impact (both benefits and risks) of the new treatment can be adequately assessed. These randomized studies are often extremely expensive to conduct because they are very resource intensive. However, they can also lack generalizability to the broader population of patients, and are often too short to identify long-term treatment effects. Furthermore, the studies are usually designed to establish efficacy and therefore are not sufficiently powered for safety outcomes.

\section{Benefits of pharmacoepidemiology in drug development}

In the preapproval drug development phase, the aim is to use pharmacoepidemiology to better understand the target population and the target indication. In this regard, it can be very helpful to identify which sections of the target population have been included in the clinical trial programme, and consequently which sections of the population may need further follow up postauthorization. Pharmacoepidemiology studies in the development period should also help to identify the major comorbidities of the underlying disease, and any relationship between the investigational treatment and these comordibities. For example, it is known that patients with rheumatoid arthritis have an underlying increased risk of cardiovascular disease, and this is important information when assessing any increased cardiovascular risk that may be 
associated with certain nonsteroidal anti-inflammatory drugs. In this regard, it is also important to understand how well the comorbidity profile of the target population matches that of the clinical trial population, because this will provide an understanding of the types of patients likely to receive the drug about whom very little is currently known. This information can be used for planning proactive pharmacovigilance, to ensure that the safety profile of the product is actively extended.

It is also possible that pharmacoepidemiology studies in the development phase can be used to explain why it is not appropriate to include certain groups of patients in the clinical trial population. One example of this of relevance to the EU would be the use of epidemiology to provide justification for requesting a waiver for a paediatric investigation plan. A number of waivers are published by the European Medicines Agency [EMA, 2010a], usually relating to classes of products or therapeutic areas. Epidemiological data to justify the applicability of a class waiver to a specific product would be a useful addition to the available information. Other specific study outcomes that may be relevant in the drug development phase include identifying the current treatment modalities, and any specific treatment pathways/guidelines. These studies may provide very useful information for the design of postauthorization studies, in particular for identifying potential sources of bias/confounding, by understanding the place that a new treatment is likely to occupy.

In general these studies are carried out by using preexisting healthcare databases, and extracting cohorts of patients with the target disease, unless literature searches can provide current, relevant, epidemiological information. In view of the recent changes in prescribing practice for many drugs, and the changing demographics of the population, it is extremely important that epidemiological information is up to date. For example, increasing prescribing of statins has led to a decrease in cardiovascular morbidity affecting a wide range of conditions, including type 2 diabetes. Therefore, any epidemiology study prior to the launch of a new hypoglycaemic agent would need to include the most recent information on health outcomes. Depending on the generalizability of the database chosen for an epidemiological study for a given indication, one study may be sufficient, or more than one may be required.

\section{Risks of pharmacoepidemiology in drug development}

Given that the majority of pharmacoepidemiology studies carried out in the preauthorization period are likely to be information gathering, and either noncomparative or disease centred studies, there are very few, if any, risks of carrying out pharmacoepidemiology studies in the preauthorization period. However, it is important that any studies carried out for the purpose of informing future studies are well designed in order to capture the important information that may be required. It is also helpful to compare the incidence rate of various comorbidities between the target population for a new drug (as identified via epidemiology studies) and the clinical trial population because this may help inform the likely differences and areas of missing knowledge between the clinical trial population and the target population, and therefore the areas for focussing additional postauthorization studies.

If such work is not carried out, then there is the risk that the target population will be misunderstood, potentially leading to unexpected safety problems, or restrictions in approvable indications.

\section{Pharmacoepidemiology postapproval}

\section{Benefits}

With the exception of drugs for orphan indications, the patient population who will be exposed to the drug in the postapproval phase will increase rapidly, from a typical clinical trial population of hundreds, to an exposed population of hundreds of thousands or even millions. It is generally accepted that the postauthorization and clinical trial populations will differ not just in terms of disease characteristics and comorbidities, but also in terms of the adherence to the treatment schedule, duration of treatment and personal characteristics that are often not well captured in clinical trial programs. Furthermore, the clinical trial and postauthorization population differences do not only apply to patients, but also to prescribers. Such differences can have an effect on the safety profile of the product as well as on efficacy, and some of these differences can be characterized by means of well designed pharmacoepidemiology studies. 
Traditionally, follow up of the safety profile of a drug in the postauthorization period relied on spontaneous reporting systems (e.g. UK Yellow Card Scheme [MHRA, 2010], FDA MedWatch Scheme [FDA, 2010]). These spontaneous reporting systems are still extremely important in postmarketing pharmacovigilance, particularly for identifying rare, serious drug effects. However, these systems rely on the healthcare professional or patient recognizing the medical condition as a drug side effect and this is less likely for side effects that may occur sometime after drug exposure, or where the effect may be an increase in the risk of a common comorbidity, such as increased risk of cardiovascular disease with COX II inhibitors. The development and implementation of ICH E2E has led to an overhaul in the expectations on drug companies in the postlicensing period with specific followup studies often requested in Japan [PMDA, 2010] and in the EU [EMA, 2010b] to ensure a more proactive approach. The overall aims of these studies are to ensure that the types of patients taking the new product are well characterized, differences between the postauthorization and clinical trial population are well understood, and any safety signals from the clinical trials are followed up.

The reasons for requesting this additional information are to ensure that a good profile of the product in real-life use is available, and that it is possible to consider any differences from the clinical trial profile.

This has clear benefits as the knowledge base on the product is extended, and any areas of concern (e.g. off-label use, patients not studied in clinical trials) can be well characterized. Obtaining this information proactively rather than reactively should help to better protect public health and build public confidence.

In order to design the best studies and identify the most relevant data sources, it is helpful to consider what level of information is known from the clinical trial program, and what additional information is expected from the postauthorization study. Together with information on estimated patient exposure, and the study objectives, these can help identify the best ways to conduct proactive pharmacovigilance [Wise et al. 2009].

\section{Risks}

Unfortunately, once a product is no longer in clinical trials, it can be difficult to find a good comparator group for pharmacoepidemiology studies for the reasons outlined above in the section on sources of data. This is not generally a concern for studies investigating demographics and prescribing trends because the observance of such trends is the reason behind the study. However, it may be a concern for studies which are considering effectiveness or safety endpoints, particularly where these may also be related to the disease being treated.

As an example, consider drug $\mathrm{X}$ used to treat cancer either first line or when drug $\mathrm{Y}$ has not been successful. Assume that the comparator group for a study of the drug in clinical practice is patients not prescribed drug $\mathrm{X}$. If deaths due to disease progression are considered as an endpoint, then it is likely that patients prescribed drug X may appear to have a worse outcome as some of these patients will already have failed on one treatment. Whilst this is an extreme and obvious example of a study design flaw, more subtle examples can occur. An example of this occurred early in postmarketing for varenicline, when a number of spontaneous reports related to psychiatric symptoms in patients taking varenicline for smoking cessation were received by regulators. This is challenging to study observationally because smokers are known to have an increased risk of certain psychiatric conditions compared with the general population, and smoking cessation is also known to have an adverse emotional effect with irritability and depression reported. Furthermore, many people have more than one attempt to stop smoking, and it was possible that people receiving varenicline were people who had already tried various other treatments.

Gunnell and colleagues [Gunnell et al. 2009] carried out a study using the GPRD. The study design needed to take these factors into account to minimize the effects of bias, and it is not straightforward to identify a suitable comparison group. Patients were categorized to the three exposure groups (varenicline, bupropion, or nicotine replacement therapy) based on the drug they were first prescribed in the follow-up period. Subsequent treatment episodes were not included in the analysis, but patients who received other smoking cessation treatments after the index prescription were identified, and 
follow up censored at the time of switching or adding products. The final results of this study did not suggest any increased risk of suicide and suicidal behaviour for people using varenicline as their first recorded smoking cessation treatment compared with people first prescribed other smoking cessation treatments.

The use of routine data sources may also complicate study design and interpretation if measures of disease severity and accurate information on confounders for all study participants are not available. These concerns become more of an issue when the potential side effect under study is related to the disease being treated and also related to the reason for choosing that specific treatment. Such 'confounding by indication' is very difficult to address in pharmacoepidemiology studies, and robust results may only be established in randomized controlled trials.

Partly as a consequence of the difficulty and expense of field studies together with the ready availability and relative low cost of database studies, pharmacoepidemiology has become a rich area for the development and application of methodologies to address the confounding issues that may arise when patients are not randomized [Suissa, 2000].

A number of different methodologies have been proposed, many of which aim to build on the traditional epidemiological designs and analyses. These include the use of propensity scores where the conditional probability of a patient receiving treatment is calculated based only on the covariates. These probabilities can then be used in a range of study designs, from direct matching, to use in a regression analysis [D'Agostino, 1998].

However, these methods can also lead to risks of misleading results, as many of these methods involve some form of matching of patients from the study product and comparator products to ensure that the groups are 'similar' in terms of index characteristics. Those who are deemed to be 'outliers' are then removed from the study. This may be acceptable if the risks of treatment are not also associated with comorbidity status, but if the risks are differential between the study and comparator product in the excluded patients, then vital information can be lost.

Furthermore, it should always be remembered that the propensity score will only contain information on the identified and measured confounders. Any unknown or unmeasured confounders will continue to affect the results. For example, if drug $\mathrm{X}$ is usually used in a hospital setting but occasionally prescribed and used in primary care, then propensity scores calculated using a primary care data source will be inaccurate because of likely misclassification of patients.

One methodology that attempts to deal with confounding whilst not removing 'outlier' patients is the use of patients as their own controls. This has been developed in the case-crossover method [Maclure, 2007] and self-controlled case series method [Whitaker et al. 2006], and such methods can provide a powerful alternative to more traditional designs. However, these designs require certain strong assumptions to be met for validity, and therefore their use needs to be carefully considered.

A further concern surrounding pharmacoepidemiology studies in the postauthorization period is the effect of stimulated reporting and publication bias. This is most familiar to those in pharmacovigilance who assess spontaneous reporting data [Sobel and Reynolds, 2008]. However, it can occur in the early stages of signal evaluation, whereby the first reports in the literature of a potential signal led to a large number of studies carried out rapidly by a range of different research groups, often using overlapping data sources, and published in a range of journals. The variety of study designs and underlying research questions can make formal synthesis of the data difficult. It may also be difficult to get studies published which show the opposite view (publication bias) and subsequent meta-analyses may suffer from a similar problem. It is not difficult to imagine that such publications may also stimulate patients to visit their doctor, and lead to additional recording of events in electronic healthcare records.

A good example of difficulties encountered in interpreting the information arising from observational studies arose when a number of observational studies raised concerns that the antifibrinolytic aprotinin, used to reduce blood loss during major cardiac surgery, may be associated with an increased risk of death. Because of the situations the drug is used in, and the likely channelling of the drug to users at high risk of perioperative complications, it was difficult to ensure that the results were not because of 
confounding by indication, whereby patients with more comorbidities were more likely to receive aprotinin in order to spare them additional blood loss and associated blood transfusion risks.

In the context of this conflicting information, a complex cost-effectiveness study was carried out in Canada (the BART study) [Fergusson et al. 2008], in which a range of antifibrinolytic products were compared in a randomized blinded controlled study. This study was not carried out by the licence holder for aprotinin. In order to ensure treatment concealment the study was designed as a triple dummy blinded study, so that all three 'interventions' were administered to all patients, with only one of the interventions being active. The study identified an increased risk of mortality in aprotinin-exposed patients, compared with patients randomized to other antifibrinolytics. This finding together with the supportive evidence from the observational studies led to the suspension of the marketing authorization for systemic formulations of aprotinin.

Such prospective randomized studies are expensive, difficult to carry out for some drugs and are therefore rare; it is noteworthy that the study was actually initiated to study cost-effectiveness safety.

\section{Discussion}

The many factors that may affect both exposure and response to drug therapy mean that the mainstay of drug development has always been the randomized controlled trial, preferably double blinded. These randomized designs reduce the impact of known and unknown confounding factors, both those affecting the physician's decision to prescribe and those affecting patient response, to ensure that different treatment groups are as similar as possible at baseline, so that the impact (both benefits and risks) of the new treatment can be adequately assessed. However, these randomized studies can lack generalizability to the broader population of patients, and are often too short to identify long-term treatment effects. Furthermore, the studies are usually designed to establish efficacy and therefore are not sufficiently powered for safety outcomes.

Pharmacoepidemiology is a rich discipline with a firm underlying methodological basis. In general, the rise of the use of pharmacoepidemiology over the previous decade can be linked with the development of formal risk management planning following on from ICH E2E, and now postauthorization studies to address drug utilization and specific safety concerns are part of the routine planning for launch of a novel product in Europe. Similar studies are carried out as part of Early Postmarketing Pharmacovigilance in Japan. This increased requirement for such studies arising predominantly from ICH E2E has been made easier by the availability of data from the use of electronic healthcare records. Such data resources mean that field studies are not always necessary, which reduces the cost of carrying out these studies. However, it is important to ensure that the study designs are rigorous and appropriate to answer the proposed study question. The use of routine data may not always be appropriate, particularly if the disease is managed or treated primarily in a hospital setting. In this case, registries may be more useful, particularly if they are disease registries enrolling patients with a range of treatments which will enable important characteristics of disease progression and management to be recorded, such as the British Society of Rheumatologists Biologics Registry [BSRBR, 2010], which was initiated to monitor the progress of patients with severe rheumatoid arthritis and other rheumatic conditions who are taking antitumour necrosis factor- $\alpha$ therapy.

The design and execution of pharmacoepidemiological studies must be addressed with care to ensure that the results are robust [Vandenbroucke, 2004a] and that bias/confounding issues are taken into account as far as possible. There are specific concerns arising in pharmacoepidemiology that general epidemiologists may be unaware of, such as issues around defining exposure and concerns over specific types of confounding. Pharmacoepidemiology studies cannot achieve such similarity between treatment groups, and therefore there is substantial room for confounding to play a role in the results from these studies. For example, it is known that patients with less stable disease are more likely to be started on new treatments than patients with stable disease, in the search for a treatment that will 'work'. However, the patients with less stable disease are also most likely to suffer progression of the disease, or other comorbidities associated with the disease. Hence any studies that do not take this information into account, while they may provide a true view of the real-life outcomes from therapy, do not 
provide a true comparison of the outcomes for patients who are treated with the new therapy compared with similar patients who are not treated.

These concerns have been discussed in the previous section, but an important consequence is the need for study authors to be moderate in their claims following from study results, and particularly for them to consider absolute as well as relative risks. Unlike other areas in which epidemiology is used, pharmacoepidemiology implicitly needs to consider benefits as well as risks of treatment, and also of patients not being treated [Vandenbroucke, 2004b]. However, relative risks are entirely unsuitable for this purpose, as understanding benefit-risk requires an understanding of the likelihood of both the benefit and of the risk Mussen, Salek and Walker.

In general, there are many more benefits than risks associated with pharmacoepidemiology. However, this is dependent on studies being conducted with care, with due regard for the quality of the data used for the study and with due regard for the potential comorbidities associated both with the disease under treatment and the choice of the specific treatment. Pharmacoepidemiological studies have the potential to provide much needed insight into treatment effects and risk factors for adverse effects once a drug is used in a larger and more diverse population than the clinical trial programme would enrol. However, when the adverse outcome under study is related to the underlying disease process and to the reason for choosing the specific drug, pharmacoepidemiology using routine data sources may not be adequate to deal with the serious confounding issues that may arise.

This may raise a question of whether observational studies should be carried out for those outcomes where it is clear that there is considerable potential for confounding that cannot be resolved. In the author's view, such studies can still be carried out, but there should be very clear and measured discussion on the implications of the results, and the level of any patient exclusions resulting from restricted analyses. The potential implications of this for internal and external validity and generalizability of the study are essential for interpreting the results.
Neither randomized controlled trials nor pharmacoepidemiology studies provide the perfect answer for drug safety, but an intelligent interpretation of results from all sources should help to improve patient safety. 'The pessimist sees difficulty in every opportunity. The optimist sees the opportunity in every difficulty' (Winston Churchill).

\section{Acknowledgements}

With grateful thanks to David Irvine and AnneRuth van Troostenburg for helpful comments and review of this article.

\section{Funding}

This article received no specific grant or funding from any public or commercial agency.

\section{Conflict of interest statement}

The author is Director of Epidemiology for Takeda Global Research and Development (Europe).

\section{References}

BSRBR (2010) British Society of Rheumatology Biologics Registry. www.medicine.manchester.ac.uk/ musculoskeletal/research/arc/clinicalepidemiology/ pharmacoepidemiology/bsrbr/ [accessed 15 December 2010].

D’Agostino Jr, R.B. (1998) Tutorial in biostatistics: Propensity score methods for bias reduction in the comparison of a treatment to a non-randomized control group. Stat Med 17: 2265-2281.

EMA (2010a) European Medicines Agency Decision on Class Waivers. Available at: www.ema.europa.eu/docs/ en_GB/document_library/Other/2009/11/

WC500011500.pdf.

EMA (2010b) European Medicines Agency Guidance on Post-Authorisation Studies. Available at: www.ema. europa.eu/docs/en_GB/document_library/ Regulatory_and_procedural_guideline/2009/10/ WC500004888.pdf [accessed 15 December 2010].

FDA (2010) AERs Database. Available at: www.fda. gov/Safety/MedWatch/HowToReport/default.htm [accessed 15 December 2010].

Fergusson, D.A., Hebert, P.C., Mazer, C.D., Fremes, S., Macadams, C., Murkin, J.M. et al. (2008) A comparison of aprotinin and lysine analogues in high-risk cardiac surgery. $N$ Engl F Med 358: 2319-2331.

Mussen, F., Salek, S. and Walker, S. (2009) A Future Framework for Benefit-Risk Appraisal of Medicines, Benefit-Risk Appraisal of Medicines: a Systematic Approach to Decision-Making, 1st edn, John Wiley \& Sons: Chichester. 
GPRD (2010) General Practice Research Database. www.gprd.com/home/ [accessed 15 December 2010].

Gunnell, D., Irvine, D., Wise, L., Davies, C. and Martin, R.M. (2009) Varenicline and suicidal behaviour: A cohort study based on data from the general practice research database. BMF 339: b3805.

ICH (2010) International Conference on Harmonisation Guideline E2e. www.ich.org/cache/compo/475-2721.html\#E2E [accessed 15 December 2010].

ISPE (2010) International Society for Pharmacoepidemiology. www.pharmacoepi.org/ [accessed 15 December 2010].

Maclure, M. (2007) 'Why me?' versus 'why now?' - differences between operational hypotheses in case-control versus case-crossover studies. Pharmacoepidemiology and Drug Safety 16: 850-853.

MHRA (2010) Yellow Card Data. Available at: http:// yellowcard.mhra.gov.uk/ [accessed 15 December 2010].

PHARMO (2010) PHARMO database. Available at: www.pharmo.nl/frames.php?htmlpage $=$ navigatie/data bases.htm [accessed 15 December 2010].
PMDA (2010) Guidance on Post-authorisation Studies. www.pmda.go.jp/english/service/outline_p.html [accessed 15 December 2010].

Sobel, R.E. and Reynolds, R.F. (2008) Integrating evidence from multiple sources to evaluate postapproval safety: an example of sildenafil citrate and cardiovascular events. Curr Med Res Opin 24: 1861-1868.

Suissa, S. (2000) Novel approaches to pharmacoepidemiology study design and statisticial analysis, In: Strom, B. (ed.). Pharmacoepidemiology, 3rd edn, John Wiley \& Sons: Chichester.

Vandenbroucke, J.P. (2004a) Benefits and harms of drug treatments. BMF 329: 2-3.

Vandenbroucke, J.P. (2004b) When are observational studies as credible as randomised trials? The Lancet 363: 1728-1731.

Whitaker, H.J., Paddy Farrington, C., Spiessens, B. and Musonda, P. (2006) Tutorial in biostatistics: the self-controlled case series method. Stat Med 25: 1768-1797.

Wise, L., Parkinson, J., Raine, J. and Breckenridge, A. (2009) New approaches to drug safety: a pharmacovigilance tool kit. Nat Rev Drug Discov 8: 779-782. 